\title{
Acción tutorial para el desarrollo de las habilidades sociales en estudiantes universitarios
}

\section{Tutorial Action for the Development of Social Skills in University Students}

\author{
Anthony Johnson Javier Napa* \\ Universidad César Vallejo, Lima, Perú \\ ORCID: https://orcid.org/0000-0002-9385-8203 \\ Héctor Raúl Santa María Relaiza \\ Universidad César Vallejo, Lima, Perú \\ ORCID: https://orcid.org/0000-0003-4598-7032 \\ Roger Pedro Norabuena Figueroa \\ Universidad Nacional Santiago Antúnez de Mayolo, Huaraz, Perú \\ ORCID: https://orcid.org/0000-0003-3731-9843

\section{Nolan Jara Jara} \\ Universidad Nacional Mayor de San Marcos, Lima, Perú. \\ ORCID: https://orcid.org/0000-0002-9467-7586
}

Recibido 25-07-18 Revisado 26-08-18 Aprobado 11-11-18 En línea 05-12-18

\begin{tabular}{lll} 
*Correspondencia & & Citar como: \\
\cline { 1 - 2 } Email: ajavier@ucv.edu.pe & & Javier, A., Santa Mría, H., Norabuena, R., \& Jara, N. (2019). \\
& Acción tutorial para el desarrollo de las habilidades sociales \\
& en estudiantes universitarios. Propósitos y \\
& Representaciones, 7(1), 185-200 Doi: \\
& http://dx.doi.org/10.20511/pyr2019.v7n1.261
\end{tabular}

(c) Universidad San Ignacio de Loyola, Vicerrectorado de Investigación, 2019.

(cc) EY-NG-ND Este artículo se distribuye bajo licencia CCBY-NC-ND 4.0 Internacional. http://creativecommons.org/licenses/by-nc-nd/4.0/). 


\section{Resumen}

La presente investigación se realizó con el propósito de diseñar talleres de tutoría en la atención de habilidades sociales poco desarrolladas de estudiantes universitarios; a través de las cuales manifiestan sus necesidades, sentimientos, preferencias, opiniones y derechos, respetando a los demás y como consecuencia se visualiza el auto reforzamiento y maximiza la probabilidad de conseguir refuerzo externo. El estudio fue abordado desde el paradigma interpretativo, enfoque cualitativo, el método proyectivo - holístico como proceso sistemático. Se recogió información diagnóstica de estudiantes y tutores, a través de entrevistas y grupos focales, centrados en las habilidades conductuales, personales y situacionales. De todo ello, se identificaron categorías y subcategorías que permitieron elegir estrategias para atender las necesidades. Como resultado, se demostró que las habilidades sociales, aspecto importante para la inserción y el desempeño laboral, no se encuentran desarrolladas a pesar de existir el acompañamiento de tutores. Asimismo, las estrategias utilizadas en las acciones tutoriales no son las adecuadas; por ende, deben ser modificadas. También, se concluyó que las instituciones de formación universitaria deben realizar procesos de planificación de tutorías previo diagnóstico.

Palabras clave: Habilidades sociales; acción tutorial; tutoría universitaria.

\section{Summary}

This research was carried out to design tutorials on the care of undeveloped social skills of university students, through which they express their needs, feelings, preferences, opinions and rights, respecting others and as a consequence self-reinforcement is observed and the probability of obtaining external reinforcement is maximized. The study was approached from the interpretive paradigm, qualitative approach, the projective - holistic method as a systematic process. Diagnostic information was collected from students and tutors, through interviews and focus groups, focused on behavioral, personal and situational skills. From all of this, categories and subcategories were identified that allowed choosing strategies to meet the needs. As a result, it was demonstrated that social skills, an important aspect for insertion and work performance, have not been developed despite the existence of the accompaniment of tutors. Also, the strategies used in the tutorial actions are not adequate; therefore, they must be modified. Also, it was concluded that university institutions must carry out planning processes for tutorials after diagnosis.

Keywords: Social Skills, Tutorial Action, University Tutoring.

\section{Introducción}

La educación superior en el Perú tiene muchos niveles de aceptación y progreso. En las últimas décadas, se ha notado un crecimiento empresarial que ha provocado el surgimiento de nuevas instituciones orientadas a brindar formación profesional. Sin embargo, son pocas las que se han preocupado en fortalecer y acompañar el crecimiento académico. Justamente, esta investigación busca diseñar estrategias en la acción tutorial para desarrollar habilidades sociales en estudiantes universitarios. Cabe recordar que, las habilidades sociales son "el conjunto de respuestas verbales y no verbales, parcialmente independientes y situacionalmente específicas, a través de las cuales un individuo expresa en un contexto interpersonal sus necesidades, sentimientos, preferencias, opiniones o derechos", tal y como lo afirmó Gismero (2010, p. 12). En base a esto, se pretende identificar respuestas estratégicas brindadas por los especialistas, no sin antes acudir a un diagnóstico para conocer las problemáticas y necesidades; de tal forma que se ofrezca un cambio en el comportamiento de los estudiantes. Esto, ya que necesitan desarrollar algunas habilidades específicas, teniendo en cuenta el entorno inmediato y los recursos utilizados. 


\section{Los talleres en la acción tutorial.}

El taller es una combinación teórica - práctica de un tema, en un determinado espacio y con la colaboración de los participantes, interpretando el aporte de Candelo, Ortiz y Unger (2003). El objetivo principal es brindar nuevas alternativas de solución a problemas específicos, utilizando la creatividad y el aporte a partir de cada experiencia personal que se concreta mediante de consensos de un número fijo de involucrados. Además, la intención de cada participante es apropiarse de nuevos conocimientos que surgen a partir de las discusiones, entre otros conceptos que se comparten en el grupo. Esto, a su vez, debe contar con algún moderador que conduzca la capacitación y establezca los objetivos claros de aprendizaje. Asimismo, la duración y la cantidad de participantes se evaluarán según algunos criterios que propongan los especialistas, teniendo en cuenta las necesidades de los capacitados. En los estudiantes, se trata de una oportunidad para seguir afianzando los conocimientos que se integran, en relación a este tipo de capacitaciones con objetivos particulares de los que buscan ser profesionales. En concordancia con ello, Reyes (1977, citado en Maya, 2007) señaló que el taller es un proceso en el que actúan los estudiantes y los docentes, comprometidos a aportar nuevos conocimientos como parte de un equipo que se integra, teniendo en cuenta la base principal que radica en unir la teoría y la práctica en esta pedagogía. Esta misma respeta una realidad social que está en constante comunicación y, que por cierto, tiende a cambiar, así como los objetivos particulares de cada participante. Partiendo de lo mencionado, podemos afirmar que la propuesta de taller realizada responde rigurosamente al diagnóstico en torno a las habilidades sociales. En ese sentido, los talleres deben ser atendidos por tutores preparados y capacitados.

Es preciso aclarar que, el tutor es una pieza fundamental en la acción tutorial. Según Candelo et al. (2003), es el personaje principal en la ejecución de un taller, porque por más que no se pueda notar en muchos casos su presencia, es él quien lo planifica, orienta y supervisa. Para ello, debe contar con ciertas habilidades y destrezas, como saber programar la actividad (teniendo claro diversas soluciones si son requeridas), tener empatía y sobre todo asertividad al momento de comunicarse con sus demás integrantes. Además de ello, manejar presupuestos y un orden administrativo que permita establecer relaciones con otras entidades. Todo esto le servirá para cumplir debidamente con la organización y ejecución de un taller, el cual necesita una persona con capacidad de liderazgo para afrontar esta dinámica.

Candelo et al. (2003) también consideró que el tutor es el encargado de que los objetivos del taller se cumplan, tomando en cuenta el proceso que debe respetarse y el contenido que se presentará. Por ende, se debe considerar diversos factores: el tema, las emociones que se generan y el método que se utiliza para la realización. Es decir, los tutores deben contar con conocimientos en base al contenido del programa, dominar el manejo de equipo y manejar una comunicación con escucha activa; de tal manera que aprenda a formular interrogantes y motive al público a compartir opiniones significativas. Debe transmitir un ambiente agradable al auditorio y no tomar posturas sobre cuestiones conflictivas. Esto se refiere al manejo de la tolerancia con los participantes. Por último, debe manejar la planificación de su tiempo y generar una retroalimentación con los participantes que dé como resultado reflexiones finales y permita cumplir las funciones de un taller.

\section{Acción tutorial y las habilidades sociales.}

El desarrollo de la tutoría se plantea a partir de la labor docente con la consigna de guiar, orientar, en varios niveles como lo personal - emocional y hasta lo académico. Para Álvarez y Castilla (2006), la acción tutorial tiene como pilar el apoyo individual y grupal en diferentes momentos que enfrenta el estudiante. En esta orientación interviene más de un docente porque el trabajo no solo lo realiza el tutor, sino que recibe la colaboración de otros profesores, quienes intervienen según corresponda la idea de manejo que se tiene. Por su parte, Arbizu, Lobato y Castillo (2005, citados en Cárdenas, 2012) afirmaron que la acción tutorial es una actividad que se trabaja a nivel grupal, ya que si bien es cierto existe una fuerte responsabilidad por parte del docente tutor, no 
podría conseguir los objetivos si es que las demás áreas de una institución no se involucran en la labor que se tiene para la mejora del estudiante y el cumplimiento de sus roles.

Cano (2012) estableció que un taller de acción tutorial debe contar con un diagnóstico, que resuma la situación de la problemática en todas sus dimensiones; una planificación que establezca las acciones de la parte operativa de los objetivos con los recursos a utilizar; una evaluación que verifique el desempeño de los logros establecidos; una sistematización que refleje la estructura planteada en base a objetivos y siguiendo un proceso; un monitoreo que visualice el constante desarrollo durante el proceso y sepa tomar decisiones eficaces en el transcurso del mismo; y una formación que plantee ciertos objetivos particulares que busquen favorecer a los participantes a partir de contenidos eficaces, tomando en cuenta los saberes anteriores de los integrantes. Para realizar la propuesta de acción tutorial es necesario conocer los parámetros establecidos en la elaboración de talleres para lograr los objetivos esperados en la atención de las necesidades.

Dentro de las diferentes posibilidades que propone la acción tutorial, Alarcón, López, Mahillo y Fernández (2014) sostuvieron que es imprescindible tener un tutor que esté involucrado con el tema académico y la debida orientación sobre ello. Esto, ya que muchas veces es necesario reforzar la parte del conocimiento. Otro aspecto que debe establecerse es el que está orientado a lo profesional, para que el estudiante vaya reconociendo sus competencias durante y después del ciclo académico. Así también, la preocupación está por el lado personal donde la asesoría tiene como objetivo brindarle estabilidad y bienestar al estudiante en el desarrollo de sus diversas actividades, específicamente el desarrollo de las habilidades sociales. Para ello, el tutor debe cumplir la función de un docente entendiendo la importancia de incorporar la reflexión crítica y una actitud de autoinspección de sus prácticas para el logro de sus objetivos (Rodríguez \& Hernández, 2018).

De acuerdo a Gismero (2000), las habilidades sociales son entendidas como:

Conjunto de respuestas verbales y no verbales, parcialmente independientes y situacionalmente específicas, a través de las cuales un individuo expresa en un contexto interpersonal sus necesidades, sentimientos, preferencias, opiniones o derechos sin ansiedad excesiva y de manera asertiva, respetando todo ello en los demás, que trae como consecuencia el autorreforzamiento y maximiza la probabilidad de conseguir refuerzo externo (p. 12).

Para Vived (2011), las habilidades sociales "son capacidades que se adquieren por medio del aprendizaje y el desarrollo, por lo que se pueden potenciar desde la escuela y desde otros contextos" (p.16). Este mismo autor afirmó que los alumnos, fundamentalmente, requieren atención y un seguimiento apropiado, durante la ejecución de los talleres.

El taller de acción tutorial, en la presente investigación, se planteó desde la perspectiva de Gismero (2010), autor que sirvió como guía para la selección de habilidades sociales mencionadas en su escala. Entre ellas tenemos la expresión de enfado o disconformidad, donde el estudiante intenta demostrar sus sentimientos en diversas situaciones, más aún cuando se enfrenta a una injusticia que normalmente deja pasar por problemas de personalidad. Sin embargo, esta expresión o la falta de ella también pueden suscitarse por dificultades o complejos de inferioridad, debido a motivos familiares o personales. Otra habilidad social es iniciar interacciones positivas con el sexo opuesto, donde se comprueba la capacidad de relacionarse con los demás con la ayuda de una buena comunicación. Pese a ello, muchas veces la falta de interacción comunicativa hace reconocer la falta de iniciativa por parte de la persona. Es más, la autoestima es responsable de muchos éxitos y fracasos académicos (Chilca, 2017).

En la habilidad social de la defensa de los propios derechos como consumidor, se destaca el hecho de reaccionar ante situaciones específicas que obligan a la persona a hacer valer derechos 
básicos. El decir no y cortar interacciones es una habilidad social que destaca la forma en cómo las personas se rehúsan a interrumpir a una situación poco agradable o una conversación que no despierta mayor interés. Además, aprender a decir no es una acción que se ve poco reflejada cuando la poca reacción y falta de carácter toman poder. De otro lado, se identificó la autoexpresión en situaciones, referida al hecho en donde la persona tiene la capacidad de dominar su comunicación verbal y no verbal; además de controlar su ansiedad en diversos momentos que enfrenta. Sin embargo, los casos opuestos también se pueden presentar poniendo en evidencia la inseguridad de sus actos. Por último, se presentó el indicador denominado hacer peticiones, que resalta la capacidad que tienen algunas personas para emitir algún pedido o realizar algún reclamo, porque se siente con la confianza y el carácter para hacerlo sin ninguna complicación. Adicionalmente, se presentó el caso contrario donde la persona se deja dominar por su timidez y no es capaz de pedir siquiera un favor por la inseguridad que lo aqueja.

\section{Método}

Esta investigación se desarrolló dentro del paradigma interpretativo, enfoque cualitativo, método de investigación proyectivo, tipo holístico. El diseño del presente estudio fue no experimental porque se orientó a observar ciertos comportamientos ya establecidos en una población determinada, sin generar una situación intencional. Por el contrario, fue apreciar un momento en donde los participantes se presentaban de forma natural.

La muestra censal estuvo conformada por 30 estudiantes de la Facultad de Ingeniería de Sistemas de la Universidad César Vallejo Lima Norte, en el año 2017. Quienes también tuvieron participación, como parte relevante, fueron 5 docentes tutores de la mencionada institución universitaria.

Se tuvo en cuenta una escala estandarizada de habilidades sociales propuestas por Gismero (2000), compuesta por 33 ítems, con 5 alternativas de respuesta, denominado EHS (Escala de habilidades sociales), cuya validez fue realizado por expertos y la confiabilidad a través del análisis confirmatorio del ítem, este instrumento sirvió para identificar las habilidades sociales no desarrolladas, luego se aplicó una entrevista a ocho estudiantes para confirmar las debilidades encontradas, confirmada la información, se realizó una entrevista a cuatro especialistas para proponer estrategias en la mejora de habilidades sociales para resolver las problemáticas expuestas en el diagnóstico de los estudiantes. A partir de la información recogida y la categorización de la misma se elabora la propuesta de acción tutorial.

\section{Resultados}

Se identificó que los estudiantes presentan habilidades sociales poco desarrolladas, problemas de expresión de emociones, temor a una figura que transmite autoridad, evaden situaciones adversas, tienen complicaciones de inferioridad y carecen de afecto en el hogar. También, hacen notar el miedo al rechazo, consternación frente a halagos, cobardía para interactuar con los demás, complicaciones para manifestar sus sentimientos y problemas para entender al otro. Asimismo, se observó que los estudiantes sí manifiestan su malestar a la otra persona, son víctimas de persuasión, tienen miedo en una confrontación grupal y cumplen con una personalidad complaciente. Es más, hay estudiantes que tienen complicaciones en la comunicación, adolecen de temperamento para decir no, son propensos a la persuasión y siempre están buscando la aceptación de los demás. Tienen dificultades para expresar y poner de relieve sus opiniones, se les complica comenzar y mantener una conversación, tienen miedo a iniciar nuevas relaciones interpersonales y poca comprensión para trabajar en conjunto. 
Tabla 1.

Resultado del diagnóstico del estudiante.

\begin{tabular}{|c|c|c|c|c|c|c|}
\hline $\begin{array}{l}\text { Expresión de enfado } \\
\text { o disconformidad }\end{array}$ & $\begin{array}{c}\text { Interacciones } \\
\text { positivas con el sexo } \\
\text { opuesto }\end{array}$ & $\begin{array}{l}\text { Defensa de lo } \\
\text { propios derech } \\
\text { como consumic }\end{array}$ & & $\begin{array}{l}\text { Decir no y cortar } \\
\text { interacciones }\end{array}$ & $\begin{array}{l}\text { Autoexpresión en } \\
\text { situaciones }\end{array}$ & Hacer peticiones \\
\hline 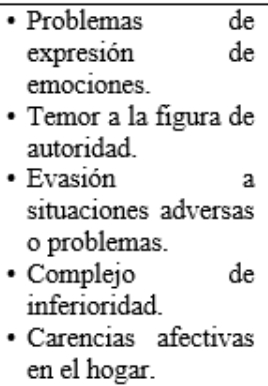 & $\begin{array}{l}\text { - Existe temor al } \\
\text { rechazo. } \\
\text { - Tendencia a } \\
\text { turbarse frente a } \\
\text { halagos. } \\
\text { - Timidez para la } \\
\text { interacción. } \\
\text { - Dificultad para } \\
\text { expresar } \\
\text { sentimientos. } \\
\text { - Dificultad para } \\
\text { comprender al otro. }\end{array}$ & $\begin{array}{l}\text { - Manifestación } \\
\text { malestar hacia } \\
\text { persona. } \\
\text { - Víctimas } \\
\text { persuasión. } \\
\text { - Temor } \\
\text { confrontamiento } \\
\text { grupal. } \\
\text { - Personalidad } \\
\text { complaciente } \\
\text { pasiva. }\end{array}$ & 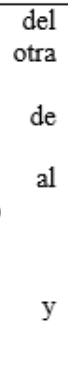 & $\begin{array}{l}\text { - Dificultad para la } \\
\text { comunicación. } \\
\text { - Falta de carácter } \\
\text { para decir no. } \\
\text { - Proclives a la } \\
\text { persuasión. } \\
\text { - Búsqueda de la } \\
\text { aprobación de los } \\
\text { demás. }\end{array}$ & $\begin{array}{l}\text {-Dificultades para } \\
\text { expresar y hacer } \\
\text { valer las opiniones. } \\
\text {-Dificultad para } \\
\text { iniciar y mantener } \\
\text { un diálogo. } \\
\text { - Temor a establecer } \\
\text { nuevas relaciones } \\
\text { sociales. } \\
\text {-Poca tolerancia para } \\
\text { trabajos en grupo. }\end{array}$ & $\begin{array}{l}\text { - Problemas de } \\
\text { inseguridad } \\
\text { (timidez } \\
\text { introversión) } \\
\text { - Sobrevalorización } \\
\text { de aptitudes y } \\
\text { cualidades. } \\
\text { - Ansiedad en la } \\
\text { expresión. }\end{array}$ \\
\hline
\end{tabular}

Tabla 2.

Resultado de estrategias propuestas por los especialistas.

\begin{tabular}{|c|c|c|c|c|c|}
\hline $\begin{array}{l}\text { Expresión de enfado } \\
\text { o disconformidad }\end{array}$ & $\begin{array}{c}\text { Interacciones } \\
\text { positivas con el sexo } \\
\text { opuesto }\end{array}$ & $\begin{array}{c}\text { Defensa de los } \\
\text { propios derechos } \\
\text { como consumidor }\end{array}$ & $\begin{array}{l}\text { Decir no y cortar } \\
\text { interacciones }\end{array}$ & $\begin{array}{l}\text { Autoexpresión en } \\
\text { situaciones }\end{array}$ & Hacer peticiones \\
\hline $\begin{array}{l}\text { - Se debe decir lo que } \\
\text { se piensa y siente. } \\
\text { - Uso de recursos } \\
\text { personales para } \\
\text { resolver conflictos. } \\
\text { - Proceso de } \\
\text { aceptación y amor } \\
\text { propio. } \\
\text { - Fortalecimiento de } \\
\text { la autoestima en el } \\
\text { núcleo familiar. }\end{array}$ & $\begin{array}{l}\text { - Debe generarse } \\
\text { confianza en sí } \\
\text { mismo. } \\
\text { - Reconocimiento de } \\
\text { capacidades y } \\
\text { habilidades. } \\
\text { - Compañerismo y } \\
\text { amistad para } \\
\text { relacionarse. } \\
\text { - Uso de la } \\
\text { comunicación } \\
\text { asertiva. } \\
\text { - Situarse en el lugar } \\
\text { del otro. }\end{array}$ & $\begin{array}{l}\text { - Atreverse a decir su } \\
\text { malestar. } \\
\text { - Capacidad de } \\
\text { negociación. } \\
\text { - Técnicas para } \\
\text { resolución de } \\
\text { problemas. } \\
\text { Técnicas para el } \\
\text { desarrollo personal. }\end{array}$ & $\begin{array}{l}\text { - Responder ante } \\
\text { situaciones } \\
\text { complicadas. } \\
\text { - Firmeza en } \\
\text { transmitir sus } \\
\text { decisiones. } \\
\text { - Evaluar y elegir la } \\
\text { mejor opción. } \\
\text { - Reconocimiento } \\
\text { del valor personal. }\end{array}$ & $\begin{array}{l}\text { - Reforzamiento de } \\
\text { los pilares de la } \\
\text { autoestima. } \\
\text { - Confianza en la } \\
\text { capacidad verbal. } \\
\text { - Salir de la zona de } \\
\text { confort. } \\
\text { - Reconocimiento de } \\
\text { las habilidades de } \\
\text { los demás. } \\
\text { - Uso de las } \\
\text { herramientas } \\
\text { comunicacionales. }\end{array}$ & $\begin{array}{l}\text { - Empoderamiento } \\
\text { de la persona. } \\
\text { - Reconocimiento } \\
\text { de las limitaciones } \\
\text { de la persona. } \\
\text { - Canalización de } \\
\text { la ansiedad. }\end{array}$ \\
\hline
\end{tabular}

Tabla 3.

Resultado de la teorización.

\begin{tabular}{|c|c|c|c|c|c|}
\hline $\begin{array}{l}\text { Expresión de enfado } \\
\text { o disconformidad }\end{array}$ & $\begin{array}{c}\text { Interacciones } \\
\text { positivas con el sexo } \\
\text { opuesto }\end{array}$ & $\begin{array}{l}\text { Defensa de los } \\
\text { propios derechos } \\
\text { como consumidor }\end{array}$ & $\begin{array}{l}\text { Decir no y cortar } \\
\text { interacciones }\end{array}$ & $\begin{array}{l}\text { Autoexpresión en } \\
\text { situaciones }\end{array}$ & Hacer peticiones \\
\hline $\begin{array}{l}\text { - Expresión y manejo } \\
\text { de emociones. } \\
\text { - Resolución } \\
\text { conflictos. } \\
\text { - Autoestima. }\end{array}$ & $\begin{array}{l}\text { - Autoestima. } \\
\text { - Expresión y manejo } \\
\text { de emociones. } \\
\text { - Empatía. }\end{array}$ & $\begin{array}{l}\text { - Asertividad. } \\
\text { - Toma de decisiones. } \\
\text { - Resolución de } \\
\text { conflictos. } \\
\text { - Identidad personal. }\end{array}$ & $\begin{array}{l}\text { - Asertividad. } \\
\text { - Toma } \\
\text { decisiones. } \\
\text { - Autoestima. }\end{array}$ & $\begin{array}{l}\text {-Autoestima. } \\
\text {-Comunicación. } \\
\text {-Toma de decisiones. } \\
\text {-Trabajo en equipo. } \\
\text {-Comunicación. }\end{array}$ & $\begin{array}{l}\text { - Autoestima. } \\
\text { - Expresión } \\
\text { manejo de } \\
\text { emociones. }\end{array}$ \\
\hline
\end{tabular}




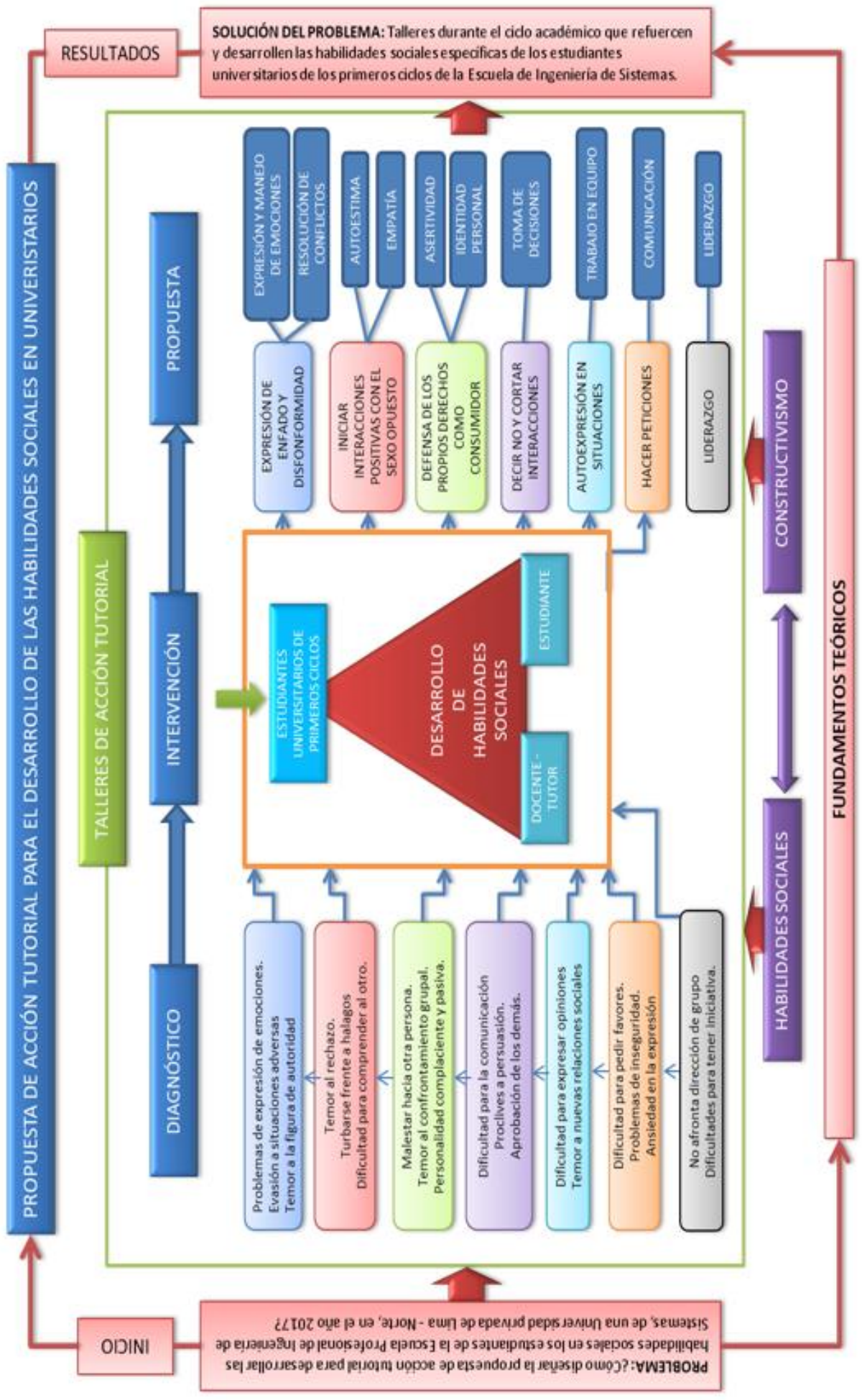

Figura 1. Propuesta de acción tutorial para el desarrollo de habilidades sociales. Fuente: Elaboración propia.

\section{Discusión}

La investigación de Hernández-Sánchez y Ortega (2015), bajo el tema "Aprendizaje Electrónico Afectivo: un modelo innovador para Desarrollar una Acción Tutorial Virtual de Naturaleza inclusiva", se consideró similar a los resultados que arrojaron el miedo al rechazo por parte de los 
estudiantes. Sin embargo, se debe tomar en cuenta que los objetivos fueron diferentes, ya que por un lado se quiso tener presente la adaptación de los estudiantes en un contexto educativo y con docentes especiales, mientras que en el estudio actual se tomó en cuenta la labor docente y especificaciones en algunas habilidades sociales.

Por su parte, Genoveva, Navarro y López (2014) propusieron "El aprendizaje de las habilidades sociales en la Universidad. Análisis de una experiencia formativa en los grados de educación social y trabajo social". En contraposición a ello, el estudio actual presentó algunos resultados que demostraban la problemática sobre la inseguridad que tienen al momento de interactuar con las demás personas, sobre todo cuando se trata de pedir algún favor. Por ello, se identificó a estudiantes que tenían dificultades por la timidez o la introversión, pero también a quienes demostraron una sobrevaloración de su personalidad que les impedía realizar acciones sencillas como pedir favores.

\section{Referencias}

Alarcón, P., López, D., Mahillo, M., \& Fernández, M. (2014). Gestión Automatizada de Tutorías. REDU: Revista de docencia universitaria, 12(2), 351-373. Doi: https://doi.org/10.4995/redu.2014.5653

Álvarez, M., \& Castilla, C. (2006). La acción tutorial: su concepción y su práctica. Madrid: Ministerio de Educación y Ciencia Editores.

Candelo, C., Ortiz, G., \& Unger, B. (2003). Hacer Talleres: Una guía práctica para capacitadores. Cali: Grafiq Editores.

Cano, A. (2012). La metodología de taller en los procesos de educación popular. Red Latinoamericana de Metodología de las Ciencias Sociales, 2(2), 22-52. Recuperado de: http://sedici.unlp.edu.ar/bitstream/handle/10915/26946/2223-3626-1-PB.pdf?sequence=1

Cárdenas, R., Terrón, M., \& Rebolledo, T. (2012). La docencia y la acción tutorial: una experiencia práctica en el ámbito universitario. Sevilla, España: I Congreso Virtual Internacional sobre Innovación Pedagógica y Praxis Educativa - Innovagogia.

Chilca, L. (2017). Autoestima, hábitos de estudio y rendimiento académico en estudiantes universitarios. Propósitos y Representaciones, 5(1), 71-127. Doi: http://dx.doi.org/10.20511/pyr2017.v5n1.145

Genoveva, R., Navarro-Segura, L., \& López, P. (2014). El Aprendizaje de las Habilidades Sociales en la Universidad. Análisis de una Experiencia Formativa en los Grados de Educación Social y Trabajo Social. Formación Universitaria, 7(4). 25-38. Doi: http://dx.doi.org/10.4067/S0718-50062014000400004

Gismero, E. (2000). EHS Escala de Habilidades Sociales. Manual. Madrid: TEA Publicaciones de Psicología Aplicada.

Hernández-Sánchez, A., \& Ortega, J. (2015). Aprendizaje Electrónico Afectivo: un modelo Innovador para desarrollar una Acción Tutorial Virtual de Naturaleza Inclusiva. Formación Universitaria, 8(2), 19-26. Doi: http://dx.doi.org/10.4067/S0718-50062015000200004

Maya, A. (2007). El taller educativo: ¿Qué es? Fundamentos, cómo organizarlo y dirigirlo, cómo evaluarlo. Bogotá: Magisterio Editorial.

Rodríguez, J., \&Hernández, K. (2018). Problematización de las prácticas docentes y contextualización de la enseñanza. Propósitos y Representaciones, 6(1), 507-541. Doi: http://dx.doi.org/10.20511/pyr2018.v6n1.211

Vived, E. (2011). Habilidades sociales, autonomía personal y autorregulación. España: Prensas Universitarias de Zaragoza. 